\title{
MRI-based neoadjuvant therapy indication in middle and low rectal cancer
}

\author{
Danihel Jr L ${ }^{1}$, Rajcok $\mathrm{M}^{1}$, Mosna $\mathrm{K}^{2}$, Belan $\mathrm{V}^{3}$, Kukucka $\mathrm{M}^{1}$, Novisedlakova $\mathrm{M}^{4}$, Oravsky $\mathrm{M}^{1}$, \\ Veres $\mathrm{P}^{5}$, Danihel Sr $\mathrm{L}^{2}$, Schnorrer $\mathrm{M}^{1}$
}

3rd Surgical Clinic, Faculty of Medicine, Comenius University in Bratislava, Slovakia. l.danihel@gmail.com

\begin{abstract}
OBJECTIVE: The aim of this study was to evaluate the indication of neoadjuvant therapy in patients with middle and low rectal cancer based on MRI examination.

BACKGROUND: In spite of noticeable advances in the diagnosis of rectal cancer, the optimal treatment remains highly debated. Current guidelines advise the use of neoadjuvant therapy in UICC stage II patients or higher. However, in clinical praxis, there is gradual implementation of new criterions and variables used in rectal cancer stage evaluation, the fact of which influences the treatment choice. The most important emerging variables taken currently into account are the distance from mesorectal fascia, circumferential resection margin, extramural venous invasion and intersphincteric plane, all of which can be evaluated using the MRI examination.

METHODS: The accuracy of MRI staging was compared with definite histopathological results from resected tumors. Patient data were prospectively collected between the years 2013 and 2018 at 3rd Surgical Clinic, Faculty of Medicine, Comenius University in Bratislava, Slovakia. Data from 101 patients were gathered and divided into two groups, according to the localization of tumor within rectum, while 9 patients were excluded from the study because of benign lesion diagnosis based upon final histopathologic evaluation.

RESULTS: In 92 evaluated patients, no significant change was noted between MRI and histopathological T-staging. However, in N-staging, significant differences were noted between preoperative MRI staging and postoperative histopathological staging.

CONCLUSION: The results of this study demonstrate inefficient preoperative lymph node staging, suggesting overtreatment of rectal cancer patients. Although the use of neoadjuvant therapy has led to great advances in modern cancer treatment, it is connected with a number of side effects and therefore should be indicated only for patients who can benefit from this treatment (Tab. 1, Fig. 3, Ref. 16). Text in PDF www.elis.sk. KEY WORDS: rectal cancer, MRI, preoperative staging, lymph node, neoadjuvant therapy.
\end{abstract}

\section{Introduction}

The incidence of rectal tumors continues to have a rising tendency in developed countries, while the age of patients at the time of diagnoses is shown to be lowering persistently. In spite of the spread of global oncology education outside healthcare professionals, rectal tumors are often diagnosed in advanced stages of disease. Statistically, the incidence of colorectal cancer comprises $10.2 \%$ of all cancer cases worldwide, namely with 1.8 million newly diagnosed cases in 2018 (55.8 \% were males; $44.2 \%$ were females) (1). In 2018, highest amount of newly diagnosed cases

${ }^{13 r d}$ Surgical Clinic, Faculty of Medicine, Comenius University in Bratislava, Slovakia, ${ }^{2}$ Institute of Pathological Anatomy, Faculty of Medicine, Comenius University in Bratislava, Slovakia, ${ }^{3}$ Dr. Magnet-Magnetic Resonance Kramáre, Bratislava, Slovakia, ${ }^{4}$ Oncology Department, University Hospital Bratislava, Slovakia, and ${ }^{5}$ Pathology Medicyt Bratislava, Slovakia

Address for correspondence: L. Danihel, MD, PhD, 3rd Surgical Clinic, Faculty of Medicine, Comenius University in Bratislava, Namestie SNP 10, SK-814 65 Bratislava, Slovakia.

Phone: +421.904331928 was noted in countries of East Asia, North America and CentralEastern Europe. However, according to the age-standardized incidence, Slovakia is the third country with highest colorectal cancer incidence, following South Korea and Hungary. From all colorectal cancers, rectal cancer comprises $38.1 \%$, while colon cancer and anal cancer comprise $59.3 \%$ and $2.6 \%$ respectively $(1,2)$.

Despite continuous medical and scientific efforts in the past decades, the exact cause of rectal cancer remains unknown and the choice of the treatment of these patients has not been united so far. The currently most argued issue regarding the rectal cancer treatment is that of the indication of neoadjuvant therapy in UICC (International Union against Cancer) stage II or higher rectal cancer patients, followed by surgical resection. Currently accepted criteria often take into account the preoperative lymph node status, even though they statistically do not yield sufficient accuracy. The aforementioned factuality resulted into the effort of developing more sensitive parameters that would allow for a more accurate preoperative staging of the disease. Among them, the most promising seem to be the measurement of distance from mesorectal fascia, assessment of circumferential resection margin 
(CRM), intersfincteric plane as well as extramural venous invasion (EMVI) (3).

A precise preoperative staging of rectal cancer diagnosed in clinical praxis by combination of endoscopic and imaging techniques plays a crucial role in the choice of adequate treatment for each patient. Main impact is currently ascribed to MRI examination, which is presently considered the most accurate imaging method in the assessment of rectal cancer. However, in spite of many advances in diagnostics of given cancer, there is still the tendency to overstage the disease. The latter overstaging takes place because the current imaging techniques do not allow to differentiate between the morphologic appearance of lymph nodes being infiltrated by tumor and those reactively changed. The main goal of modern rectal cancer treatment is to select a group of patients that would benefit from primary surgical resection treatment without neoadjuvant therapy, and thus improving their quality of life without increasing the risk of local recurrence of the disease.

This study focuses on analyzing the accuracy of preoperative staging of middle and low rectal cancer based on the comparison between MRI staging and histopathological staging of the resected tumor tissue, and subsequently on evaluating the potential primary surgical resection treatment indication based on new parameters implemented in preoperative MRI diagnostics.

\section{Patients and methods}

This prospective study included exclusively only patients with middle and low rectal cancer treated at 3rd Surgical Clinic, Faculty of Medicine, Comenius University in Bratislava, Slovakia between September 2013 and September 2018. The study was executed after ethics committee approval. Overall, 101 patients with primary surgical resection treatment were included, 47 of which were of female gender and 54 were male. Patients were furthermore subdivided into two groups according to the distance of tumor from anus, namely the first group included patients with middle rectal tumors (6-12 cm from anus) while the second group comprised those with low rectal tumors (0-6 cm from anus).

All selected patients had to fulfill a number of strict criteria based on MRI examination, particularly the distance of tumor from mesorectal fascia being greater than $5 \mathrm{~mm}$, T1 to T3b tumor stage, free intersphincteric plane, free CRM and negative EMVI, while the lymph nodes were not taken into account when determining the stage of disease. All MRI examinations were carried out at the same establishment (Dr.Magnet), while using the same MRI machine to minimalize the bias of the examination. Patients that met the given criteria were indicated to primary surgical resection of the tumor, which was followed by histopathological examination of the resected tissue. The quality of surgical treatment was assessed histopathologically, based on the distance of tumor from mesorectal fascia and examination of at least 12 lymph nodes.

Collected data were evaluated statistically using Fisher's exact test to determine differences in the representation of individual data, while the difference in data representation obtained from two different approaches was based on Chi-square test. The results were considered statistically significant when $\mathrm{p}$ value was $<0.05$.

\section{Results}

The average age of patients included in the study was 69.5 years. Out of 101 patients initially enrolled, 9 patients who were initially diagnosed with invasive carcinoma on MRI and presented with high-grade dysplasia on preoperative histological examination had to be excluded due to verification of benignity of rectal lesion based on final histological evaluation. Even though these patients were not included in final statistical analysis, they could be considered as part of the group that had been overstaged upon MRI examination.

The remaining 92 patients were divided into two groups according to the level of rectum affected. Group 1 comprised patients with tumor in the middle third of rectum - 62 patients (67\%), Group 2 contained patients with lesions located in the lower third of rectum - 30 patients (33\%).

\section{T-staging}

The T-staging evaluation of 92 patients' lesions yielded overall success rate of preoperative MRI 68\% (63 patients) when compared with pathological T-staging evaluation based on the examination of resected specimen. Overstimated T-staging (overstaging) was noted in $20 \%$ of cases (18 patients) and underestimated T-staging (understaging) was found in $12 \%$ (11 patients). Statistical significance in overall T-staging accuracy was not observed $(p>0.06)$.

The analysis of T-staging targeted on the location of tumor showed an accuracy of $73 \% 45$ patients) and $60 \%$ (18 patients) in patients with middle rectal tumor and low rectal tumor, respectively. Overstaging was noticed mainly in T2 and T3 tumors. It was noted in 10 patients (16\%) with middle rectal tumors, and 8 patients (27\%) or those with low rectal tumors. On the other hand, understaging was captured in $11 \%$ of patients with middle

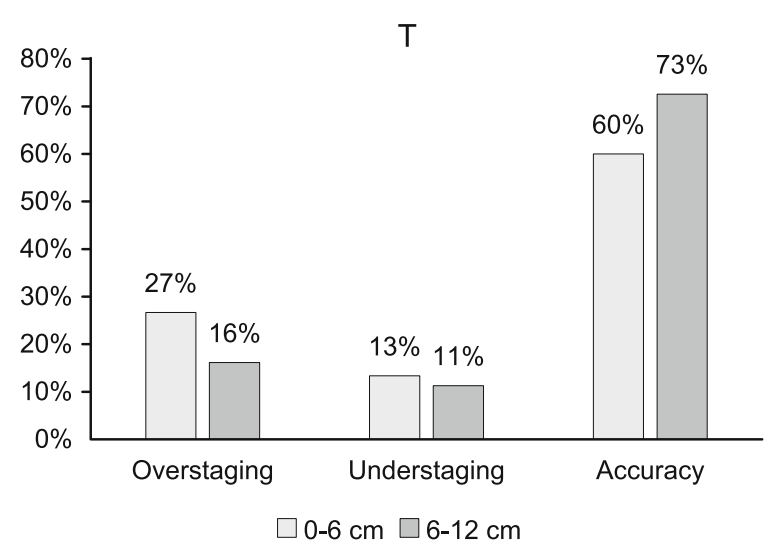

Fig. 1. T-staging accuracy of preoperative MRI examination in low and middle rectal tumors. 


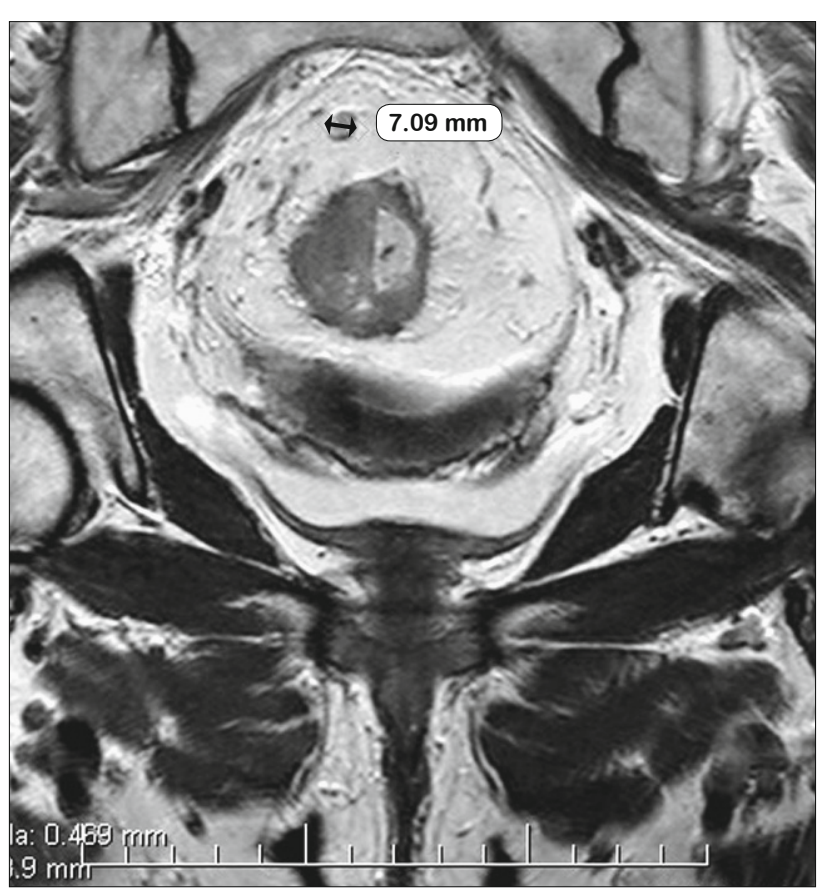

Fig. 2. Positive lymph node by MRI.

rectal tumors (7 patients) and $13 \%$ of those with low rectal tumors (4 patients). The difference in T-staging in any rectal compartment in the investigated group of patients was not significant (p > 0.08) (Fig. 1).

\section{$N$-staging}

The topic of preoperative $\mathrm{N}$-staging of tumors remains widely debated since none of the currently available imagining techniques can safely differentiate lymph nodes with tumor infiltration from those with a reactive change only (Fig. 2). When comparing MRI-based N-staging with histopathological N-staging, conformity was noted in $39 \%$ of cases (36 patients). Understaging was

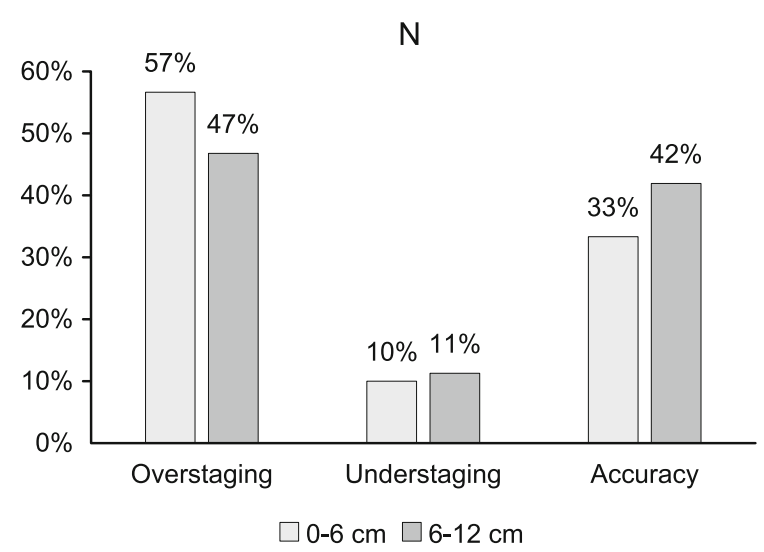

Fig. 3. N-staging accuracy of preoperative MRI examination in low and middle rectal tumors. present in $11 \%$ of cases (10 patients), however, overstaging was present in as many as $50 \%$ of investigated cases (46 patients), out of which the complete negativity of lymph nodes was proven by histological examination in 38 cases.

The accuracy of the evaluation of N-staging based on the localization of tumor was revealed to be best in cases with middle rectal tumors, in whom it reached $42 \%$ (26 patients). A somewhat lower precision was noted in patients with low rectal tumors, in whom it reached $33 \%$ (10 patients). The biggest issue in lymph node evaluation is represented by preoperative overstaging. In this study, the overstaging of lymph nodes was detected in $47 \%$ of cases with middle rectal carcinomas (29 patients), and in as many as $57 \%$ cases with low rectal tumors (17 patients). When considering the importance of therapy choice, the degree of accuracy achieved by lymph node evaluation in rectal tumor staging is inefficient. On the other hand, the understaging of lymph nodes in groups with low rectal tumor and middle rectal tumor was noted in markedly fewer cases, namely only in $10 \%$ (3 cases) and 11\% (7 patients), respectively. In the investigated group of patients there was a significant difference in $\mathrm{N}$-staging in low rectal compartment $(\mathrm{p}<0.04)$ (Fig. 3).

\section{Mesorectal fascia distance}

The distance of tumor from mesorectal fascia was more than $5 \mathrm{~mm}$ in all included patients. The consecutive histopathological analysis did not show infiltration of mesorectal fascia in any of the 92 patients. The exact values of the distance of tumors from mesorectal fascia were obtained only later throughout the study. To prevent misinterpretation of the results, further analysis of accuracy of preoperative measurements of mesorectal distances was not pursued.

\section{Discussion}

Nowadays, the treatment of rectal cancer remains relatively heterogeneous which requires a multidisciplinary approach and individual assessment of each case. The accuracy of preoperative staging continues to be essential in selecting the optimal treatment management for each patient. The imaging techniques most utilized in preoperative rectal cancer staging still include endoscopic ultrasonography (EUS), CT and MRI. Nowaday, the most important imaging examination in rectal cancer diagnosis is considered to be MRI, which accounts for the most accurate visual display of pelvic tumors. Amongst other variables, the current MRI examination of rectal tumors utilizes also circumferential resection margin (CRM) assessment as well as measurement of exact distance of tumor form mesorectal fascia. A 5-mm distance of tumor from mesorectal fascia on the MRI reading correlates with that of $1 \mathrm{~mm}$ in histopathological examination (4). According to the Al-Sukhni meta-analysis, the assessment of CRM on MRI examination yields a $77 \%$ sensitivity and $94 \%$ specificity (5).

The overall results of this study prove the need of MRI examination in high-volume medical centers. This is due to initial inaccuracy in evaluation of border $\mathrm{T} 2$ and $\mathrm{T} 3$ tumors. This inaccuracy 
is mainly accredited to the desmoplastic reaction which considerably complicates radiologic evaluation due to dense fibrous tissue around the tumor. With the passing time and more experiences, we were able to successfully minimalize this factor.

The biggest differences regarding rectal cancer treatment are observed in neoadjuvant treatment indication. In the future, the indication criteria of neoadjuvant therapy in rectal cancer have to be reconsidered. This is essential in order to prevent overtreatment in the selected group of patients. According to the currently recognized guidelines, patients in UICC stage I cancer are indicated to primary surgical resection, while the patients in higher stages are recommended to be administered with previous neoadjuvant treatment. New information is emerging, which is just slowly and very cautiously being adapted by various oncological societies. Since the benefits of short-course or classical neoadjuvant treatment were not confirmed in cases with T1 and T2/N0 tumors, the German S3-Guidelines advise primary surgical resection in allthese patients (6). Latter randomized studies evaluating neoadjuvant treatment in locally advanced rectal tumors did not include patients in the first stages of disease. Swedish trial results proved lower local recurrence in patients with neoadjuvant therapy ( $4.5 \%$ vs $14 \%$ after 13 years), however, at the time of the study, the total mesorectal excision was not at use (9). Contrary to the latter studies, those conducted in Netherlands and Great Britain have not concluded the difference between patients with and without neoadjuvant treatment to be significant (1\% vs $3 \%$ after 10 years and $1.9 \%$ vs $2.8 \%$ after 3 years) $(6,10)$. On the other hand, for UICC stage II and III tumors in middle and low rectum, it is recommended to apply neoadjuvant treatment followed by surgical resection (11). T1 and T2 tumors with uncertain lymph node involvement represent an exception to this rule, as well as T3a/b tumors without definite lymph node metastases (5). From our point of view, since it is not always possible to state with certainty the possible lymph node involvement, this recommendation should be flexible. ESMO classification is in this matter considerably more resolute with suggestion of primary tumor resection in stage II and III patients meeting the established ,good prognosis“ criterions and anticipated good quality total mesorectal excision (12) (Tab. 1).

Tab. 1. Parameters of good and poor prognoses of rectal cancer patients according to The Magnetic Resonance Imaging and Rectal Cancer European Equivalence (MERCURY) Study 2014 (Modification after Taylor FG, 2014).

\begin{tabular}{lcc}
\hline MRI & Good prognosis & Poor prognosis \\
\hline CRM1 & $>1 \mathrm{~mm}$ & $<1 \mathrm{~mm}$ \\
\hline Tumors of lower & Intersphincteric & Intersphincteric \\
rectum $<5 \mathrm{~cm}$ & plane without & plane with tumor \\
from anus & tumor infiltration & infiltration \\
\hline T-staging & T1/T2 & T3c $>5 \mathrm{~mm}$ \\
& T3a $<1 \mathrm{~mm}$ & T4 \\
& T3b $<1-5 \mathrm{~mm}$ & \\
\hline EMVI2 & Negative & Positive \\
\hline N-staging & Any stage & Any stage
\end{tabular}

${ }^{1}$ Circumferential resection margin, ${ }^{2}$ Extramural venous invasion
Present-day recommendations prefer the management of upper rectal tumors to be consistent with colon cancer. This study focuses on middle and low rectal tumors, since in upper rectal tumors, there was no proof of advantage of MRI examination sensibility in comparison with CT examination. The results of this study unambiguously conclude significant differences in MRI based N-staging and definite histopathological N-staging, which proves a notable inaccuracy in preoperative lymph-node staging in rectal cancer patients. On the other hand, the deviation of histopathological T-staging from preoperative T-staging showed to be insignificant.

Similar studies conclude the potential benefit of new MRI parameters in rectal cancer assessment, which could constitute the basis of neoadjuvant therapy indication in these patients. Results of the Magnetic Resonance Imaging and Rectal Cancer European Equivalence (MERCURY) Study, that included patients selected according the novel MRI criteria (T1-T3b, free intersphincteric plane, free CRM, without EMVI) undertaking primary surgical resection without neoadjuvant treatment, showed a 3-year recurrence rate of $3.3 \%$ (13). Other aspects leading to a more accurate preoperative diagnosis of rectal cancer originate from the introduction of advanced MRI imaging techniques such as dynamic contrast-enhanced and diffusion-weighted sequences (14-16).

\section{Conclusion}

Magnetic resonance is currently considered the most precise modality in rectal cancer diagnosis and evaluation. Despite undeniable improvement of MRI technology over the past years, it is still essential to search for new possible parameters that would lead to more exact preoperative staging and increased quality of life of patients in the preoperative period.

\section{References}

1. International Agency for Research on Cancer. New Global Cancer Data: Globocan 2018. (Online) September 2018. ( Cited: June 3, 2019) https://www.uicc.org/new-global-cancer-data-globocan-2018

2. Arnold D. WHO Klassifikation 2010 für den unteren Gastrointestinaltrakt. Der Pathologe 2016; 32 (S2): 326-331.

3. Karpitschka M, Graser A. Bildgebung und Diagnostik zur Planung des operativen Eingriffs. 2-11. In: Kreis ME, Straßburg J (Eds). Moderne Chirurgie des Rektumkarzinoms. Berlin: Springer, 2015.

4. Beets Tan RG, Beets GL, Vliegen RF et al. Accuracy of magnetic resonance imaging in prediction of tumor free resection margin in rectal cancer surgery. Lancet 2001; 357 (9255): 497-504.

5. Al-Sukhni E, Milot L, Fruitman M et al. Diagnostic accuracy of MRI for assessment of $\mathrm{T}$ category, lymph node metastases and circumferential resection margin involvement in patients with rectal cancer: a systematic review and meta-analysis. Ann Surg Oncol 2012; 19 (7): 2212-2223.

6. van Gijn W, Marijnen CA, Nagtegaal ID et al. Preoperative radiotherapy combined with total mesorectal excision for resectable rectal cancer: 12-year follow-up of the multicentre, randomised controlled TME trial. Lancet Oncol 2011; 12 (6): 575-582. 
7. Bosset JF et al. Fluorouracil-based adjuvant chemotherapy after preoperative chemoradiotherapy in rectal cancer: long-term results of the EORTC 22921 randomised study. Lancet Oncol 2014; 15 (2): 184-190.

8. Gerard JP et al. Preoperative radiotherapy with or without concurrent fluorouracil and leucovorin in T3-4 rectal cancers: results of FFCD 9203. J Clin Oncol 2006; 24 (28): 4620-4625.

9. Folkesson J, Birgisson H, Pahlman L, Cedermark B, Glimelius B, Gunnarson U. Swedish Rectal Cancer Trial: Long Lasting Benefits From Radiotherapy on Survival and Local Recurrence Rate. J Clin Oncol 2005; 23 (24): 5644-5650.

10. Sebag-Montefiore D, Stephens RJ, Steele R et al. Preoperative radiotherapy versus selective postoperative chemoradiotherapy in patients with rectal cancer (MRC CR07 and NCIC-CTG C016): a multicentre, randomised trial. Lancet 2009; 373 (9666): 811-820.

11. Leitlinienprogramm Onkologie. S3-Leitlinie Kolorektales Karzinom. Version 2.1| Januar 2019 (Online) May 2019 https://www.awmf. org/uploads/tx_szleitlinien/021-007OLl_S3_Kolorektales-KarzinomKRK_2019-01.pdf

12. Glynne-Jones R, Wyrwicz L, Tiret E , Brown G, Rödel C, Cervantes A, Arnold D. Rectal Cancer: ESMO Clinical Practice Guidelines (Online) May 2019 https://www.esmo.org/Guidelines/GastrointestinalCancers/Rectal-Cancer
13. Taylor FG, Quirke P, Heald RJ et al. Magnetic resonance imaging in rectal cancer. European Equivalence Study Group., Preoperative magnetic resonance imaging assessment of circumferential resection margin predicts disease-free survival and local recurrence: 5-year follow-up results of the MERCURY study. J Clin Oncol 2014; 32 (1): 34-43.

14. Kim SH, Lee JY, Lee JM, Han JK, Choi BI. Apparent diffusion coefficient for evaluating tumour response to neoadjuvant chemoradiation therapy for locally advanced rectal cancer. Eur Radiol 2011; 21 (5): 987-995. https://doi. org/10.1007/s00330-010-1989-y

15. Sun YS, Zhang XP, Tang L, Ji JF, Gu J, Cai Y, Zhang XY. Locally advanced rectal carcinoma treated with preoperative chemotherapy and radiation therapy: preliminary analysis of diffusion-weighted MR imaging for early detection of tumor histopathologic downstaging. Radiology 2010; 254 (1): 170 -178. https://doi.org/10.1148/radiol.2541082230

16. Engin G, Sharifov R, Gural Z et al. Can diffusion-weighted MRI determine complete responders after neoadjuvant chemoradiation for locally advanced rectal cancer? Diagn Interv Radiol 2012; 18 (6): 574-581. https://doi.org/10.4261/1305-3825. DIR.5755-12

Received June 19, 2019. Accepted July 4, 2019. 